\title{
An Energy-Balanced Routing Algorithm Based On Forward Aware Factor for Machine-To-Machine Communication Networks
}

\author{
Prasad Challa \\ Scholar in Department of CSE, JNTUA, Ananthapuramu, Andhra Pradesh, India \\ chprasad18@gmail.com \\ Dr. B Eswara Reddy \\ Professor in Department of CSE, JNTUA, Ananthapuramu, Andhra Pradesh, India \\ eswar.cse@jntua.ac.in
}

\begin{abstract}
Machine-to-Machine Communication (M2M), sometimes also entitled as Machine-to-Machine Communication Area Networks which has been a dynamic research area above the past few years and it is an essential ingredient of Engineering and Industrial Applications (EIA). The sensor nodes in the network are limited in energy, therefore, communication ability also restricted. So, in this case, this appears particularly significant for the development and designs direction finding the protocol for the broadcasting of the sensing data successfully. For the conservation of energy, a novel approach is preferred that is an Energy-Balanced Routing Algorithm based on the Forward-Aware Factor (FAFEBRA). The new concept is introduced in FAF-EBRA is hop is a segment of the path between starting place and target. The hop count refers to the number of intermediate devices through which data must pass between source and destination. In the method of FAF, the next-hop node chosen as per the perception of communication link weight and the forward energy density and additionally local topology reconstruction mechanism is designed for load-balance among the nodes. The FAF-EBRA is evaluated with LEACH \& EEUC and the results obtained through the experiments that FAF-EBRA better performance in terms of the consumption of the energy, which prolongs the network lifetime and gives assurance to maintain high Quality of Service of M2M Communication area networks.
\end{abstract}

Keywords: Energy Balanced, Forward-Aware Factor, Engineering and Industrial Application, Machine-toMachine Communications.

\section{Introduction}

The Machine-to-Machine communication is an autonomous network system of wireless and it is organized with a vast number of micro sensors with limited energy in the stream of a lot of Engineering and industrial applications (EIA). Now, M2M is extensive acts as a useful communication technology to put together both the home applications and communication in the field applications of engineering and as well as industry. A power supply is a limited one in the sensor networks. Consequently, the design of the shape of a local-area network or other communications system, a direction-finding algorithm is vital and a key to work to study an extensive level M2M communication system. In M2M controlling of topology and routing design, it needs to balance the utilizing of energy, connectivity, and multiple mechanisms are applied. The majority of the networks of EIA are independent of time role, and scope, converge to a similar architecture and so pollsters tried to make a unified replica for complex networks in the last decades. It is outstanding that remote feeler systems are a self- alliance framework with a remote system composed of the sum of strength constrained the miniaturized degree of feelers under the flag of Engineering and Industrial Application (EIA) [1], [2].

At present days, M2M network is a successful medium that is largely utilized to coordinate the data universe of EIA [3]-[6]. Each sensor center consisting of a sensor and a switch, and its capacity to register, accumulation of stockpile is in the limit, correspondence capability, and the supply of the power is restricted. In this way, it delineates of the topology of the system, steering calculation, and convention is the most vital and key work to examine the extensive scale M2M correspondence framework [7]-[12].

As of late, it is outstanding that remote feeler systems are a self- alliance framework with a remote system composed by the sum of strength constrained miniaturized degree of feelers under the flag of Engineering and Industrial Application (EIA) [13] [14]. A huge segment of the actual systems of EIA is autonomous of their age, capability, and also, it unites to designs [15], [16], therefore analysts attempted to construct a brought together an innovative replica in the complicated systems. 
In [17], Erdös, as well as Rényi, suggest ER arbitrary diagram show given great chart hypothesis and measurable material science.

In [18], the global property of the complex structure of the system is set up by Watts and Strogatz. In [18], the worldwide things of the multifaceted system are set up by Watts and Strogatz, set up WS world organization. In [19], Barabási and Albert build the BA show, uncovers the without a level of scale. In [20], the BBV model is manufactured by Barrat, et al., this model explains the quality of associations and modifications of accumulation of quality into the novel thought. In the present scenario, the model of BBV is mainly utilized to investigate the genuinely complicated systems, for example, a joint effort of the researcher put in order (SCN) and overall airplane fatal point of the system (WWAN) [21]- [23]. Like SCN and WWAN are dissimilar nodes and its structures of their group (bunches) in M2M communication, critical nodes (group heads) have a huge number of organizations than normal nodes.

Many nodes look into "energy opening" which explains that the stream of information on every association fluctuates broadly in WSAN in light of these assorted separations to the sink node.

Therefore, it is not a proper reasonable one to speak to an association as associated ("1") or connectionless ("0"). Besides, global data controlled in M2M of EIA sensors deal data their "neighborhood world". By and large, the weighted system and neighborhood hypothesis are fitting to show M2M of EIA. When compared to the LEACH and EEUC the proposed system FAF-EBRA gives the best results to increase the lifetime of the M2M communication network.

\section{Related Work}

\subsection{Low Energy Adaptive Cluster Head (LEACH) Procedure}

LEACH procedure is a well-known WSN tree based routing algorithm in which nodes are having the capability for reorganization of local topology. In the LEACH routing protocol, the nodes sort out themselves into local cluster, the routing process divided into set up stage and steady state stage, in the setup stage the clusters are formed and in steady state stage data is transferred from the nodes to the sink node under [1]-[3].In the set up stage, every node pick a random number in the between 0 and 1, if this number is not up to the threshold value $T(n)$, the node will broad cast as the cluster leader the non-cluster leader chooses the cluster leader with higher signal strength and joins the group, and the cluster leader node receives the data from all the nodes and sends the data to the remote sink node[4]- [7]. The threshold value is denoted with T (n) is given as

$$
T(n)= \begin{cases}\frac{p}{\left(1-p * r \bmod \left(\frac{1}{p}\right)\right)}, & n \in G \\ 0, & \text { else }\end{cases}
$$

Where $p$ is the rate of the cluster leader account for entire sensor node, present figure of the round, and $r$ represents the current number of communication round $G$ denotes the set of nodes that have not part of the cluster group.

In the steady stage state, data is sending from the sensor nodes to the cluster leader and on to the sink node. The new cluster leader is elected for each communication round by this way the load is balanced among the sensor nodes present in the M2M communication area network.

\subsection{Energy-Efficient Uneven Clustering (EEUC) Procedure}

EEUC is an cluster routing procedure in which tentative cluster leaders use uneven competition ranges $R_{c}$ to construct clusters of the sizes of uneven [8]-[11].

$$
R_{c}=\left(1-c \frac{d_{\max }-d(i, \operatorname{Sink})}{d_{\max }-d_{\min }}\right) R_{c}^{0}
$$

Where $d_{\max }$ and $d_{\min }$ are the furthest distance and the nearest distance between sensor nodes the to the sink node, $d(i, \operatorname{Sink})$ is the distance between and node $i$ and the sink, and $R_{c}^{0}$ is the maximum of the competition ranges (which is a fixed value, but $R_{c}$ is a variable value), the value of $c$ is between 0 and 1 which depend on the competition ranges, in other works it indicates that the node have smaller value closure to the sink node and larger value far to the sink node. Therefore the cluster leader selected based the closure to the sink node. 


\section{BBV Weighted-Network Model and Local-World Theory}

Based on our work relating to research, developing the topology of BBV replica could be separated into the stages of four [20].

1. Initialization: In the early of the network consists of nodes and a smaller number of edges $\left(w=w_{0}\right)$. Here, $w_{0}$ is the original weight, and changeable limitation weight $\mathrm{w}$ is $w_{0}$.

2. Topology Growth: At each case in point step, a join through the edges at the access network.

3. Preferential count: Nodes are giving an advantage to close-by edges in step 2) with probability $p_{n \rightarrow i}$ as follows

$$
p_{n \rightarrow i}=\frac{S_{i}}{\sum_{j} S_{j}}
$$

Wherever $n \rightarrow i$ denotes node $\mathrm{n}$ to node $\mathrm{i}$, the strength of the apex $S_{j}$ define as the summary of edge masses related to it: $s_{i}=\sum_{j \in n(i)} w_{i j}, w_{i j}$ Is the heaviness between node and Node $\mathrm{j}, \mathrm{n}(\mathrm{i})$ is a set of neighbored nodules (directly connect to node). The node strength $S_{j}$ is similar.

4. Update effectiveness as well as masses (as obtainable in Fig.1). The totaling of the edge(n, i) not vary the strong idea of the node, besides, it also alters the weights between and its neighbors

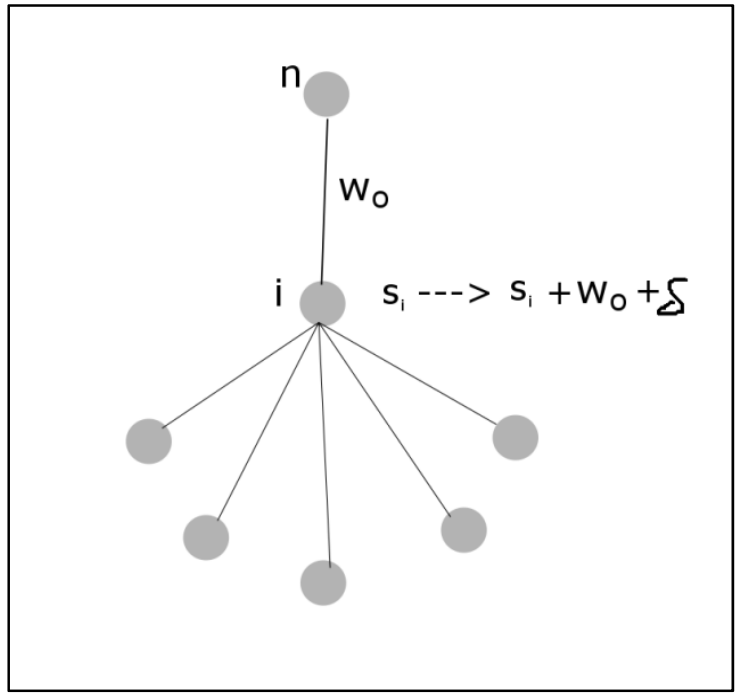

Fig.1. Change the node strength

$$
W_{i j} \rightarrow W_{i j}+\Delta W_{i j}
$$

$$
\text { where } \quad \Delta W_{i j}=\delta \frac{W_{i j}}{S_{j}}
$$

Here, $\delta$ is an increment of either positive or negative. Subsequent updated repeating the steps 2)-4), until the developing is completed. As an assumption, let in $w_{0}=1$, when $t \rightarrow \infty$, the distribution of edge weight is

Distribution of node degree

$$
\begin{aligned}
& P(w) \sim w^{-\alpha} \\
& P(k) \sim k^{-\gamma} \\
& P(s) \sim s^{-\gamma}
\end{aligned}
$$

At this point $\delta$ is an increment of either an optimistic or bad. The developing model of the system is scheduled by Li and Chen [10]. These instructions show that whereas in the definite system, a nodule could hardly attach to the singular collection of the nodules rather than whichever nodule in the whole system. M nodes are designated as arbitrarily among the current bulges as the limited biosphere of the original nodule and the preferred connection prospects are clear as 
Here, $\mathrm{M}\left(\mathrm{M} \leq N_{0}\right)$ nodes among the existing arrange as the neighborhood universe of new device node collected with original edges and is a historical factor. In the BBV demonstration, current centers from the entire arrangement are elected to limit with the new center, which is not feasible in a Wireless Sensor Actuator Networks (WSAN) of EIA because of the restricted communication transmission range and energy of sensors.

$$
\begin{aligned}
& P_{\text {local }}(n \rightarrow i)=p^{1}(1 \epsilon \text { local }- \text { world }) \frac{k_{i}}{\sum_{j} k_{j}} \\
& \text { where } p^{1}(1 \epsilon \text { local }- \text { world })=\frac{M}{\left(N_{0}+t\right)}
\end{aligned}
$$

Thus the local world theory is required, that is to state, $n$ can only connect with the sensors within a particular range. In SCN, the similar approach is used among scientists to cooperate with other persons who effort in a similar state or intercity. Also in the WWAN, the flight distance is often shorter than the greatest rage of a plane, which can be observed as the examples of the local world topology. [10-13].

\section{Forward-Aware Factor Energy Balanced Routing Algorithm(FAF -EBRA)}

In the proposed FAF-EBRA we study the details analysis of the data capturing in M2M communication area network and quantify the forward transmission area and forward energy density which are factors for the edge weights. In this way the load among the nodes adjusted and thus improves the network lifetime of the communication network.

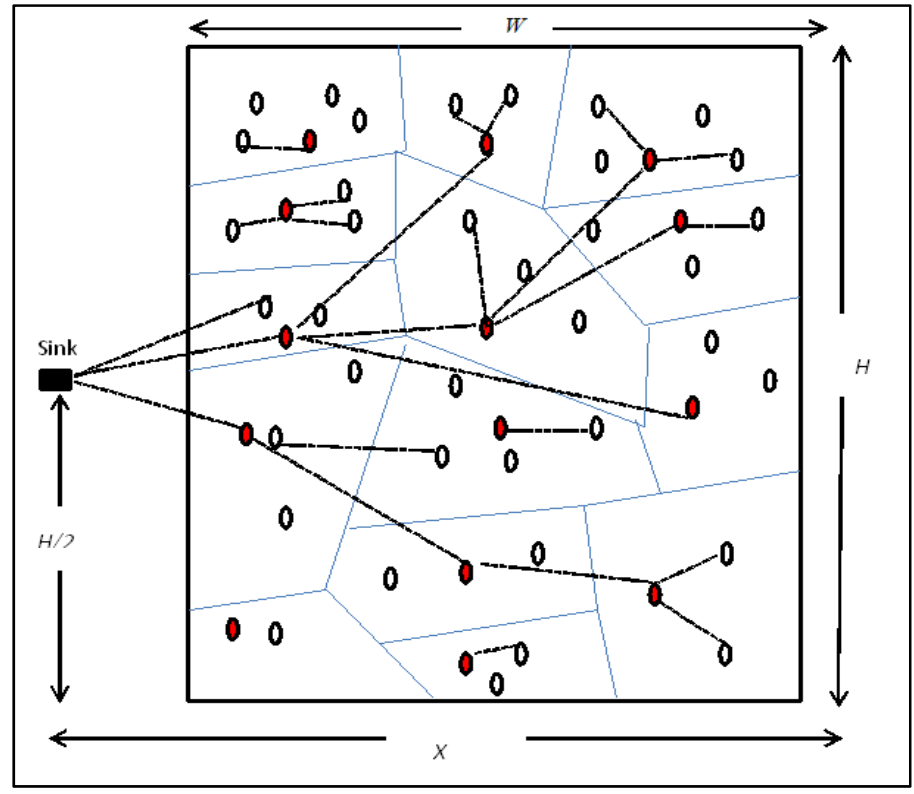

Fig.2. Allocation plan of sink and sensor node

\subsection{Network Model}

As seemed in Fig. 2, assume device nodes are randomly distributed in a $W \times H$ rectangular sensing field. The communication network is divided into several clusters and all the nodes present in the cluster should transfer the data packets to the cluster leader node. The cluster leader is the native dominant node and consequently sends the data packets to the sink node. The descriptions and definitions are as follows.

1) All the sensor nodes are isomorphic, and they have constrained abilities to compute and communicate and store the captured data. The sensors are defined as $V=\left\{V_{1}, V_{2}, \ldots V_{n}\right\}$ where $\mathrm{n}$ is the total number of sensors. The nodes are identified by their index number $i \in\{1,2, \ldots n\}$, here $i$ indicates the identifier of the node.

2) The initial energy of the sensor nodes is same for all nodes and it is denoted by $E_{0}$, and the nodes stop communicating if the energy is exhausted completely. The sink node is having continuous power supply. We assumed that all the nodes in the network are stationary.

3) Nodes can have the ability to adjust the transmission range. The sink node can broadcast the messages to all the sensor nodes in the communication are network. The receiver signal strength indicates the distance between signal source and the destination. The cluster leader nodes nodes are not chosen at the beginning of the commutation, despite they spring up during the topology evolution. 
The energy model of the network is free space model [8]. The energy consumed for transmitting a l-bit data packet over the distance $d$ is given as

$$
\begin{gathered}
E_{T x}(l, d)=E_{T x-e l e c}(l)+E_{T x-a m p}(l, d) \\
=\left\{\begin{array}{l}
l E_{\text {elec }}+l \varepsilon f_{S} d^{2}, d<d_{0} \\
l E_{\text {elec }}+l \varepsilon m p d^{4}, d<d_{0}
\end{array}\right.
\end{gathered}
$$

$$
\text { Where } \quad d_{0}=\sqrt{\frac{e_{f s}^{2}}{e_{m p}}}
$$

Where $\varepsilon_{f s}, \varepsilon_{m p}$ represent the energy coefficients. The energy consumption of sensor nodes while receiving the data packets ss

$$
E_{R x}(l)=E_{R x-e l e c}(l)=l E_{\text {elec }}
$$

Where $E_{\text {elec }}$ value is energy consumed for receiving the 1-bit data. When the distance to transmit the data is more than the threshold value $d_{0}$, the energy consumption increases sharply, therefore the maximum communication range of the sensor nodes is set to $d_{0}$.

As shown in the Fig.2, the distance of the Sink is $d(i$, sink), is given as

$$
d(i, \operatorname{Sink}) \in\left(X, \sqrt{\left((H / 2)^{2}+(X+W)^{2}\right)}\right)
$$

In the same Fig.2., the range of communication can be measured to shape a topology so with jagged bunches, when the collection head, the range of the bunch is $R_{\text {opt }}(i)$ :

$$
\begin{gathered}
R_{\text {opt }}(i)=f_{1}(d(i, \operatorname{Sink})) \\
\text { where } f_{1}(d(i, \operatorname{Sink})) \text { is a function for } d(i, \operatorname{Sink}) \\
f_{1}(d(i, \operatorname{Sink})) \in\left(0, d_{0}\right)
\end{gathered}
$$

Definition 3: At a time $t$, The edge weight between $i$ and $\mathrm{j}$ sensor nodes is given by

$$
w_{i j}(t)=\frac{\varsigma\left(E_{i}(t) E_{j}(t)\right)^{\psi}}{\left(d(i, j)^{2}\right)^{\eta}\left(T_{i j}(t)\right)^{\xi}}
$$

Where $\zeta, \psi, \eta$ and $\xi$ are non-negative coefficients, $E_{i}(t)$ and $E_{j}(t)$ are the residual energies of node I and $\mathrm{j}$ at the time $t, d(i, j)$ is the distance between two nodes, and $T_{i j}(t)$ is the data movement of the control link of the communication. Set the distance from $i$ to Sink farther than $j$ and then

$$
T_{i j}(t) \sim f_{2}(d(i, \operatorname{Sink}), t)=\frac{t}{\left(d(i, \operatorname{Sin} k)^{2}\right)}
$$

Where $f_{2}(d(i, \sin k), t)$ is a function of lessening of $d(i, s i n k)$ and with a cumulative function of time $t$. The sum of data is minor when the edge-finish node is outside away from the nodule of the sink.

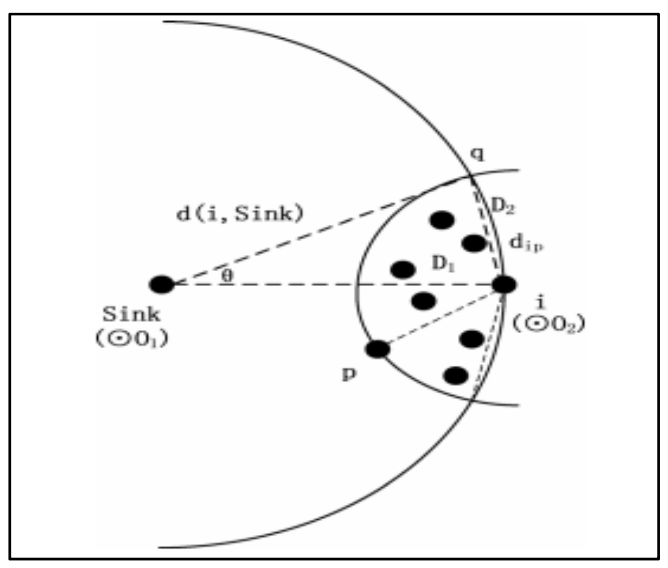

Fig.3. Forward Transmission Area of node $i$ 
As the time goes on the number of communication rounds increases by this the quantity of data present in the network grows simultaneously. In this explanation, the weight of the edge $w_{i j}$ represents the capacity of the communication which is present in the eq. (18), when, $d(i, j)$ is long, the data transmission tends to selects a link of short-distance. Similarly, when $T_{i j}(t)$ is larger, the communication link is busy shows busy, the data transmission chooses the low-load link. Here the energy plays a key role in edge weight, when the residual energy of nodes $i$ and $j$ is adequate, (the edge from $i$ to $j$ ) is stronger for the transfer of data packets.

The nodes forward energy density is denoted as $F E D(i, t)$ and defined as the

$$
F E D(i, t)=\frac{\sum_{j \in F T A(i)} E_{j}(t)}{S_{F T A}(i)}
$$

Where $E_{j}(t)$ is the energy value of node $\mathrm{j}$ at time $\mathrm{t}$ and $\sum_{j \in F T A(i)} E_{j}(t)$ denotes the all of the neighbors energy combined in FTA(i).

The Forward Aware Factor for the communication is defined as the link between the nodes $\mathrm{i}$ and $\mathrm{j}$ given as

$$
F A F(i j)=\alpha \frac{F E D(j)}{\sum_{j \in F T A(i)} F E D(j)}+\beta \frac{w_{i j}}{\sum_{j \in F T A(i)} w_{i j}}
$$

Where $\sum_{j \in F T A(i)} F E D(j)$ is all the neighbors FAF combined in FTA(i). The weight of edge $\left(w_{i j}\right)$ is defined in (16),$\sum_{j \in F T A(i)} w_{i j}$ is all of the edge weights combined that I has in FTA. $\alpha$ and $\beta$ are the positive harmonic constants and

$$
\alpha+\beta=1
$$

\subsection{Design of the FAF-EBRA}

FAF-EBRA is used for the large-scale M2M Communication networks where the nodes are stationary and captures the data from all the sensors to the sink node. In (18), he first term denotes the FED of the possible next-hop neighbors into consideration, which means the means the ability to transmit the data packets. The second term considers the weight of the transmit link, which can be used to select the next hop directly. The edge weight includes key parameters such as the energy, distance and load of sensor nodes which are listed in the Table-1.

Table-1: Routing Parameter of nodes

\begin{tabular}{|c|c|}
\hline Field Name & Significance \\
\hline Neighbor_id & Unique identifier of each neighbor \\
\hline Energy_id & Energy identifier of each neighbor \\
\hline FED_id & FED identifier of each neighbor \\
\hline Distance_id & Distance identifier of each neighbor \\
\hline
\end{tabular}

The FAF-EBRA is designed in seven stages as follows

1. Define FTA ( $i$ ) all of the probable next-hop nodes of node $i$. First, take $d_{0}$ as transmitting radius, find the set of all of the nodes that have neighbors of node $i$, and denoted by the $N^{l}(i)$. Choose the nodes that are closure to the sink node than node $i$ which constitutes all the set of possible nest hop nodes and the furthest node determined by FTA(i).

2. Define $F T A(j)$ and $S F T A(j)$ of each possible next-hop node. Determine the FTA $(j)$ as we defined in FTA(i). Plug and the furthest distance between $\mathrm{j}$ and sink in FTA and the distance between $\mathrm{j}$ and Sink and get $\operatorname{SFT} A(j)$.

3. Calculate $F E D(j)$ of each possible next-hop node. Plug the nodes' energy into (19) and get FED (j).

4. Calculate the weight of edges between $i$ and every node as indicated by (16).

5. Substitute the values of 3) and 4) into (16) and compute FAF of every possible transmit link. Select the next-hop node according to

$$
j=\max _{j}[F A F(i j)]
$$

6. If there is no node closure to the sink I in $N^{1}(i)$, directly compare FAF of all of the nodes in $N^{1}(i)$, and choose the nest-hop node according to (20). If there is no node in $N^{1}(i)$, I will be increases the transmitting power of the node to get the longer radius than $d_{0}$ until connected with another node or I will be abandon the data packet. 
7. If Sink is among the forward transmitting nodes, i will be transmitting the data directly to Sink. And accomplish the procedure.

In FAF-EBRA, the routing list structure of nodes is showing as the in Table-1. The information of the table can guarantee all the parameters FAF-EBRA needed. The communication launch node can calculate the weight of edge between neighbors. Neighbors can get their own FED. It avoids the communication launch node doing all of the algorithms. Thus, each node's memory should storage its own ID, real time energy, distance to the Sink, and FED at any moment, which could be feedback to launch node quickly.

\subsection{Local Topology Reorganization}

In the actual routing process the nodes with greater signal strength will have the more communication link and results in faster energy utilization. The whole communication system cannot work under the same topology routing structure. A topology reorganization mechanism of the cluster leader rotation of LEACH algorithm is required.

The whole $\mathrm{M} 2 \mathrm{M}$ area network information is limited, and global topology change may influence the information perception, the global change caused by energy unbalanced area is waste of energy to energy balanced area, so the only local topology reorganization mechanism is needed. This paper proposed an additional procedure for the local topology reorganization.

The different steps in the local topology modifications given below,

1. In FAF-EBRA, every time node $i$ completes its data transmission, checks the point strength of the nexthop node $j$. If it is less than the average value of all the nodes strengths in FTA then the local topology reorganization mechanism is launched in node $i^{\prime}$ FTA.

2. Before going to the reconfiguration of the local topology, remove the link between $\mathrm{i}$ and $\mathrm{j}$, remove $\mathrm{j}$, from FTA(i), and get a new set $F T A^{1}(i)$ then reconnect in $F T A^{1}(i)$. and the function of reconnect possibility is given as

$$
p_{i \rightarrow j}=\frac{S_{j}}{\sum_{j \in F T A^{1}(i)} S_{j}}
$$

3. The nodule detached in 2) can be possible next-hop node when the next transmission is finished, and the revocation of the edge does not affect the possible reorganization. The nodes real-time strength is needed to calculate the sum of strength.

\section{Results}

The real M2M Communication networks are mostly complex systems that contain lots of nodes and connections. If a complex network is weighted, the weight not only represents existence of the link between nodes, but also describes the property and intensity of the connection. In Scientific Collaboration Networks (SCN), weights represent the frequency of cooperation between scientists, and in WWAN, weights represent the number of available seats in flights between two airports. Based on our research work, an energy-balanced routing algorithm FAF-EBRM based on forward aware factor is tested. In FAF-EBRA, the next-hop node is selected according to the awareness of link-weight and forward energy density, furthermore, a spontaneous reconstruction mechanism for local topology is designed additionally

In SCN, masses represent to the reappearance of teamwork amongst investigators, and in WWAN, masses represent the number of nearby seats in airlifts between binary air terminals. In light of our job assessment, energy is adjusted according to the directing the relevant technique FAF-PBRM in light of forwarding aware element is endeavor. In FAF-EBRA, the subsequent jump node is selected as per the awareness of the weight of the link and the density of forwarding energy; also, a spontaneous renovation of the native topology device is intended. Before testing many imitations [4], [5], [22], the two purposes in (17) and (19) will be quantized $R_{c}(i)$. Is intended as:

$$
R_{c}(i)-f_{1}(d(i . \operatorname{Sink}))=\left(1-c \frac{d(i . \operatorname{Sink})-X}{\sqrt{\left((H / 2)^{2}+(X+W)^{2}\right)}-X}\right) d_{0}
$$

Where $c=0.5, d_{0}=87 \mathrm{~m}, X=50 \mathrm{~m}, W=H=200 \mathrm{~m}$ which is verified arbitrarily from folder use as fresh information. Edge weight is stated as in (18)

$$
w_{i j}(t)=\frac{\varsigma\left(E_{i}(t) E_{j}(t)\right)^{\psi}}{\left(d(i, j)^{2}\right)^{\eta}\left(T_{i j}(t)\right)^{\xi}}
$$

Assumed and is become from topology construction utilizing truthful apparatus. How would we select centers from amongst the nodes? It is as indicated by arbitrary choice has given the possibility of the hypothesis. A related guideline is also identified from that. The transference of node degree, quality and weight of the edge comes into sight in 
Furthermore, the information in the hypothetical from the investigation by the mathematical vision of the concern hypothesis is dissimilarity and the information test commencing practice situations in the figures and after taking one of the practices in the situations.

M2M Communication networks could be deliberated as gauge free biased schemes which reproduce their current information and active character beneath the excellent of Broadband Internet of Cars (BIOC). Now the arrangement of BIOV and WSANs are broadly utilized as a division through online well-being read-through and safety measurements and alarming movement of the vehicles, delightful run of the carrying, and point improvement in the crossing point. By making use of the sensors of the vehicles with vast implementation, for instance, heat devices and ultraviolet devices are breaking down the pounding took from the radar and additionally its recurrence changes to maintain a strategic distance from impact mischance's and give security cautioning.

The specialized types of gear utilized by us are Crossbow, MicaZ, Iris, and the examination schemes use via us are NS-3, OMNeT++, and ATEMU. These specialized types of gear and the test systems specified above are regularly utilized by our examination gathering. As appeared in Fig. 6, trial dissemination of IA is steady with hypothetical appropriations, take after control rule and prove a "tail", which are the important qualities of sans gauge schemes. As seemed in Fig. 6, the likelihood of $\mathrm{p}(\mathrm{k})=1$.

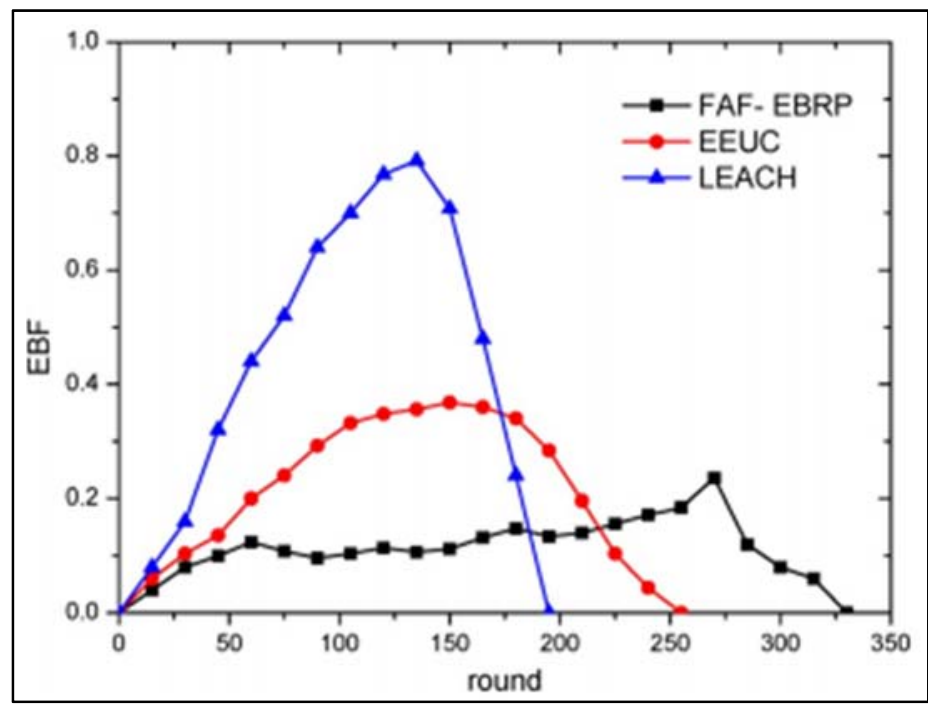

Fig.4. Comparison of EBF

Is 0.5 , represents that about part of the sensors has one interface for correspondence, which moves forward the information to the subsequent bounce node of EIA. The chance that a scheme has a wide amount of nationals (grade is bigger than 100) is small, watching that dominant centers are in the underground bounded by all devices, then the number of correspondence connections associated with one focal node is restricted.

Truth is told, similarly, as we specified over, the quantity of neighbors relies on upon taking after parameters: number of nodes, arrangement region, methodology, and radio connection run. For instance, if we convey routinely 400 devices on $200 \mathrm{~m}$ four-sided part and result in the radio connecting up to $39 \mathrm{~m}$, the number of neighbors will be equal to 8 . The association of theoretical review is alike as Fig. 6 , consequently it is ignored. Each arrangement of exploratory information has their errors, and the grade of their mistake is howl 5\% in light of our many dimension work as well as examination.

We think about LEACH, EEUC, and FAF-EBRA by three parameters: energy adjusted element (EBF), number of last-surviving nodes (NLN) and network lifetime, packet reception relation (PRR). To count the adjust of energy utilization of direction-finding conventions, EBF remains characterized as the standard deviation of the considerable number of nodes' remaining energy

$$
E B F=\sqrt{\frac{1}{N} \sum_{i=1}^{N}\left[E_{i}(t)-E_{\text {avg }}(t)\right]^{2}}
$$

Where $\mathrm{N}$ is an integer of nodes of the whole network, $E_{i}(t)$ is the node $i$ residual energy at time $\mathrm{t}$ and $E_{\text {avg }}(t)$ is the average value of remaining energy of all the nodes. FL is uncomplicatedly recognized with NLN; the FL description and requirement of is varied under different circumstances, as a few need not required passing node. 
In our examination point of view, it required considering FL of two states, one is time from the system starts to the principal passing of the nodes, and the other is time from the system start to a large portion of the nodes dead. PRR implies the proportion of the information that sinks gotten to the information that the sink should be gotten. PRR can quantify M2M network circumstance naturally.

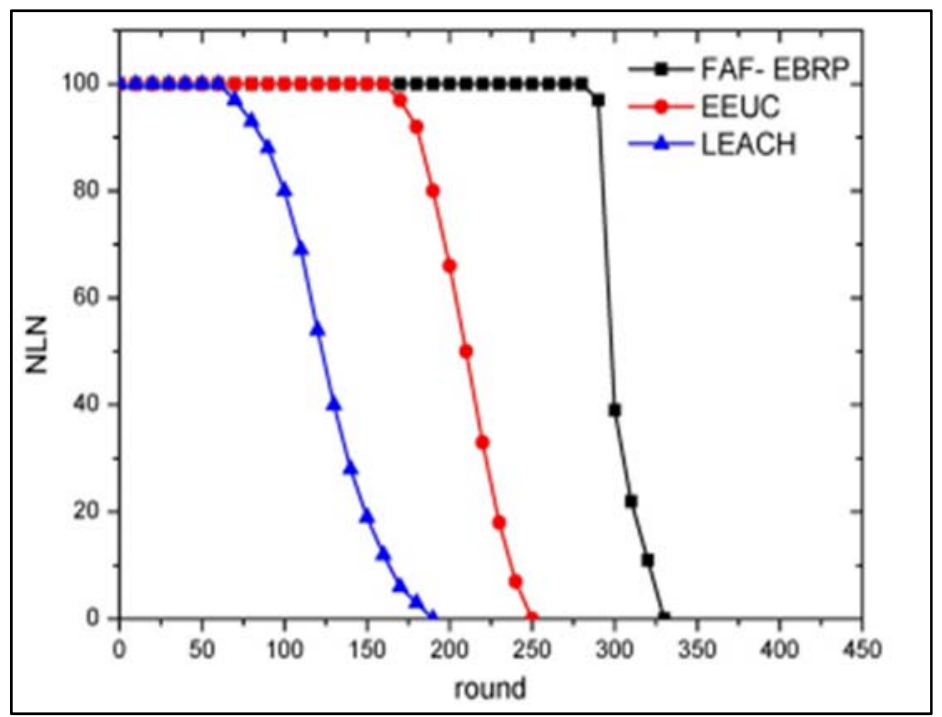

Fig.5. Contrast of NLN

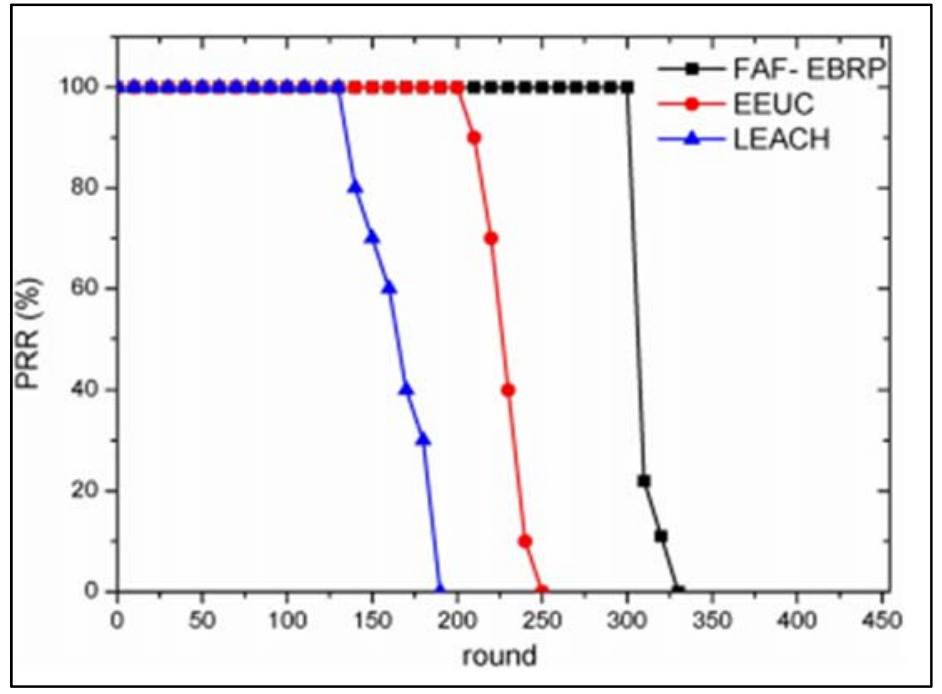

Fig.6. Comparison of PRR

In this particular article of the research paper, we set to make easy information is uneven, and the normal parcels developing rate is given to test the implementation of every conference. To think about three conventions helpfully and naturally, FAF-EBRA likewise utilizes the time round level.

Fig. 4, Fig.5 and Fig. 6 make obvious that the EBF, NLN and PRR of three conventions in the test of 350 rounds respectively. In Fig.4, the EBF of FAF-EBRA increases very marginally at the initial instant and keep ups a constant condition before encircling 250, then amplify a little time and move toward reverse to 0 as the energy of the entire system is utilizing up.

In Figure 3, the prime passing of FAF-EBRA center go roundup in anticipation of Round 300, and the technique of the nodes' termination of either quick or late. In Fig. 6 the PRR of FAF-EBRA keeps 100\% proportion for 300 rounds and the decrease arrange representing a little extent. From the outcomes of the above, we can observe that FAF-EBRA execution rate is higher than LEACH and EEUC, which adjusts the energy utilization, drags out the capacity of life span and make certain high level of QoS, (for example, EnergyBalanced, Long-Surviving, Packets Reception Radio of M2M communication networks. 


\section{Conclusion}

In this paper we proposed energy balanced routing algorithm FAF-EBRA, in this protocol the next-hop node is chosen according to the link weight and forward energy density. Furthermore we designed a local topology reconstruction mechanism. In the Simulation, FAF-EBRA is having better performance in terms of energy consumption and network lifetime against LEACH and EEUC, and test come about demonstrate that FAFEBRA outflanks LEACH and EEUC, which adjusts the energy utilization, delays the capacity lifetime, and ensures high QoS of M2M communication area network. In addition, they demonstrate that the disseminations of node degree, quality, and edge weight take after power law and speak to "tail," so the topology has strength and adaptation to internal failure, lessens the likelihood collapse and the synchronization is upgraded of use of EIA failure, and promotions the synchronization of M2M communication of industrial applications.

\section{References}

[1] L. Barabási, "Scale-free networks: A decade and beyond," Science, vol. 325, no. 5939, pp. 412-413, 2009.

[2] D. G. Zhang and X. D. Zhang, "Design and implementation of embedded un-interruptible power supply system (EUPSS) for webbased mobile application,” Enterprise Inf. Syst., vol. 6, no. 4, pp. 473-489, 2012.

[3] W. B. Heinzelman, A. P. Chandrakasan, and H. Balakrishnan, "An application-specific protocol architecture for wireless sensor networks," IEEE Trans. Wireless Commun., vol. 1, no. 4, pp. 660-670, Oct. 2002.

[4] I. K. Samaras and G. D. Hassapis, "A modified DPWS protocol stack for 6LoWPAN-based wireless sensor networks," IEEE Trans. Ind. Inf., vol. 9, no. 1, pp. 209-217, Feb. 2013.

[5] D. G. Zhang, "A new approach and system for attentive mobile learning based on seamless migration," Appl. Intell., vol. 36, no. 1, pp. 75-89, 2012.

[6] D. G. Zhang, "Web-based seamless migration for task-oriented nomadic service," Int. J. Distance E-Learning Technol., vol. 4, no. 3, pp. $108-115,2006$

[7] Q. J. Chen, S. S. Kanhere, and M. Hassan, "Analysis of per-node traffic load in multi-hop wireless sensor networks," IEEE Trans. Wireless Commun., vol. 8, no. 2, pp. 958-967, Apr. 2009.

[8] J. Jin et al., "Handling inelastic traffic in wireless sensor networks," IEEE Trans. Sel. Areas Commun., vol. 28, no. 7, pp. 1105-1115, Jul. 2010.

[9] D. G. Zhang and X. J. Kang, "A novel image de-noising method based on spherical coordinates system," EURASIP J. Adv. Signal Process., vol. 1, p. 110, 2012.

[10] X. Li and G. R. Chen, “A local-world evolving network model,” Physica A, vol. 328, no. 1-2, pp. 274-286, 2003.

[11] A. S. Ruela et al., "Evolutionary design of wireless sensor networks based on complex networks[A]," in Proc. 5th Int. Conf. Intell. Sensors, Sensor Netw., Melbourne, Victoria, Australia, 2009, pp. 237-242.

[12] J. Aweya, "Technique for differential timing transfer over packet networks," IEEE Trans. Ind. Inf., vol. 9, no. 1, pp. 325-336, Feb. 2013.

[13] C.T.Cheng, C. K. Tse, and F. C. M. Lau, "A clustering algorithm for wireless sensor networks based on social insect colonies," IEEE Sensors J., vol. 11, no. 3, pp. 711-721, Mar. 2011

[14] H. B. Zhang and H. Shen, "Energy-efficient beaconless geographic routing in wireless sensor networks," IEEE Trans. Parallel Distrib. Syst., vol. 21, no. 6, pp. 881-896, Jun. 2010.

[15] S. Vural and E. Ekici, "On multihop distances in wireless sensor networks with random node locations," IEEE Trans. Mobile Computing, vol. 9, no. 4, pp. 540-s552, Apr. 2010.

[16] F. V. C. Martins and E. G. Garrano, "A hybrid multiobjective evolutionary approach for improving the performance of wireless sensor networks," IEEE Sensors J., vol. 11, no. 3, pp. 545-554, Mar. 2011.

[17] P. Erdös and A. Rényi, "On the evolution of random graphs,” Math. Inst. Hungarian Acad. Sci., vol. 5, pp. 60-67, 1960.

[18] D. J. Watts and S. H. Strogatz, "Collective dynamics of small-world networks," Nature, vol. 393, no. 6684, pp. 440-442, 1998.

[19] A. L. Barabási and R. Albert, "Emergence of scaling in random networks," Science, vol. 286, no. 5439, pp. 509-512, 1999.

[20] A. Barrat, M. Barthélemy, and A. Vespignani, "Modeling the evolution of weighted networks," Phys. Rev. E, vol. 70, no. 6, pp. 1-13, 2004.

[21] A. Barrat et al., "The architecture of complex weighted networks," PNAS, vol. 101, no. 11, pp. 3747-3752, 2004.

[22] D. G. Zhang, "A new medium access control protocol based on perceived data reliability and spatial correlation in wireless sensor network," Comput, Electr. Eng., vol. 38, no. 3, pp. 694-702, 2012. 